\title{
Calidad web en medios digitales: revisión bibliográfica sobre métodos e indicadores de evaluación general y atributos de confianza
}

\section{Website quality in digital media: literature review on general evaluation methods and indicators and reliability attributes}

Alejandro Morales-Vargas. Universitat Pompeu Fabra. España. Universidad de Chile. Chile. amorales@uchile.c1

$[\mathrm{CV}] \bigcirc \mathrm{G} / \mathrm{R}$

Rafael Pedraza-Jiménez. Universitat Pompeu Fabra. España.

rafael.pedraza@upf.edu

$[\mathrm{CV}](\mathrm{G}) \mathrm{O}$

Lluís Codina. Universitat Pompeu Fabra. España.

lluis.codina@upf.edu

$[\mathrm{CV}]$ (1) $\mathrm{C}$

Este trabajo forma parte del proyecto "Narración interactiva y visibilidad digital en el documental interactivo y el periodismo estructurado". RTI2018-095714-B-C21 (MICINN/FEDER), Ministerio de Ciencia, Innovación y Universidades (España). Inicio: 01/01/2019. Término: 31/12/2021

También cuenta con el apoyo de la Agencia Nacional de Investigación y Desarrollo (ANID) de Chile, a través del programa Becas Chile para Doctorado en el Extranjero. Inicio: 15/10/2017. Término: 15/10/2021

Cómo citar este artículo / Referencia normalizada

Morales-Vargas, A., Pedraza-Jiménez, R. y Codina, L. (2022). Calidad web en medios digitales: revisión bibliográfica sobre métodos e indicadores de evaluación general y atributos de confianza. Revista Latina de Comunicación Social, 80, 39-63. https://www.doi.org/10.4185/RLCS-2022-1515

\section{RESUMEN}

Introducción: En el contexto de la pérdida de confianza hacia los contenidos en internet, la desinformación, las noticias falsas y la crisis financiera que afecta a la prensa a escala global, se revisan los estudios que proponen instrumentos dedicados a evaluar los sitios web de los medios digitales. Objetivos: Identificar y caracterizar los trabajos focalizados en el análisis de cibermedios, examinar sus métodos e indicadores, y revisar si estos consideran atributos de fiabilidad. Metodología: Se examina la producción científica sobre calidad web mediante una revisión bibliográfica sistematizada sobre 83 publicaciones recuperadas desde las principales bases de datos. También se detectan parámetros de calidad en general y menciones a aspectos web con implicaciones éticas o sociales. Resultados y discusión: Se constata que las herramientas específicas para medir la calidad web de los medios digitales son escasas y que entre los métodos de evaluación predomina el análisis experto mediante inspección heurística por sobre los estudios de usuario. El 
parámetro más estudiado es la interactividad, entendida como espacio de participación, seguida por la usabilidad o facilidad de uso, la arquitectura de información y el contenido, entre otros. Además, se detectan muy pocas menciones a asuntos deontológicos -como enlaces engañosos, protección de datos o corrección de informaciones- y casi nulas referencias a directrices, estándares y políticas internacionales. Conclusiones: Numerosos trabajos abordan indicadores específicos de los medios digitales, pero existen pocos protocolos de análisis para los sitios web que los sustentan. Hay un amplio margen de mejora en los estudios de la calidad web en cibermedios, en cuanto a considerar no solo asuntos técnicos sino también parámetros propios del rigor periodístico y la credibilidad informativa.

PALABRAS CLAVE: Calidad web; medios digitales; cibermedios; sitios web; métodos de evaluación; confiabilidad, desinformación.

\begin{abstract}
Introduction: In the context of the loss of confidence towards Internet content, misinformation, fake news and the financial crisis affecting the press globally, the studies that propose instruments dedicated to evaluating digital media websites are reviewed. Objectives: Identify and characterize the works focused on digital media, examine their methodologies and indicators, and check if they consider reliability attributes among them. Methodology: Scientific production on web quality is examined through a systematic review on 83 articles retrieved from the main databases, detecting parameters of web quality in general, and mentions of web aspects with ethical or social implications. Results and discussion: As part of the findings, it has been found that specific tools for the evaluation of digital media are scarce and that among the evaluation methods, expert analysis through heuristic inspection predominates over user studies. The most studied parameter is interactivity, understood as a space for participation, followed by usability, accessibility and personalization, among others. In addition, there are very few references to deontological matters and almost no references to international guidelines, standards and policies. Conclusions: Numerous studies address specific indicators of digital media, but there are few analysis protocols for websites that support them. There is, therefore, a wide margin of improvement in the studies of website quality in digital media, incorporating not only technical issues but also considering parameters of journalistic rigor and informative credibility.
\end{abstract}

KEYWORDS: Website quality; digital media; websites; evaluation methods; reliability; trustfulness; misinformation.

\title{
CONTENIDO
}

1. Introducción. 2. Metodología. 3. Resultados y discusión. 3.1. Estudios y casos 3.2. Protocolos de análisis 3.3. Métodos y herramientas 3.4. Parámetros e indicadores 3.5. Atributos de confianza 4. Conclusiones. 5. Referencias 6. Currículum Vitae

\section{Introducción}

A más de tres décadas desde la invención de la web, esta es hoy el espacio de comunicación más utilizado del mundo (Internet Live Stats, 2021). Mientras el uso de los medios tradicionales como fuente informativa ha decrecido en los últimos años a escala global, en sus versiones digitales se mantiene o aumenta. De hecho, tanto el consumo de prensa online como de redes sociales se ha incrementado sustancialmente en la mayoría de los países, según el Digital News Report (Newman et al., 2021). La versión 2021 de este informe del Reuters Institute añade que más de un $80 \%$ de los usuarios lee noticias en línea y casi tres cuartas partes lo hace a través de un teléfono móvil inteligente. 
Ahora bien, de acuerdo con este mismo informe, si ya existía inquietud por el fenómeno de la desinformación, con la aparición de la pandemia de la COVID-19 esta ha crecido. Un 58\% de los encuestados siente preocupación con respecto a qué noticias son verdaderas y cuáles informaciones son engañosas en internet (Newman et al., 2021).

Ello ha redoblado la necesidad de un periodismo riguroso y confiable, que pueda tanto informar como educar, dado lo permeables que somos a la desinformación. El mismo reporte, pero de 2018, indica que la mayoría de las personas opinan que son los directivos de los sitios web de noticias (75\%) y de las plataformas sociales $(71 \%)$ quienes tienen la mayor responsabilidad de solucionar el problema de las fake news o noticias falsas (Newman et al., 2018).

Por ello, es relevante que los sitios web de la prensa digital, los llamados cibermedios (Díaz Noci y Salaverría, 2003), apliquen criterios de calidad no solo en su contenido periodístico y estándares informativos (Rivas-de-Roca et al., 2020; Romero-Rodríguez et al., 2016), sino también en la organización, navegación, diseño visual y facilidad de uso de su interfaz. La razón es que estos aspectos son decisivos tanto en la credibilidad del sitio como en la visibilidad de las noticias.

Frente a esta preocupación, podemos encontrar respuestas en la práctica que se ocupa de la evaluación de la calidad web, la que apareció con el surgimiento de los sitios web y que con los años se ha convertido en un importante e interdisciplinario campo de estudio. Es así como, tanto desde el ámbito académico como el profesional, se han generado numerosas propuestas para el análisis de los sitios web, expresadas en publicaciones científicas y documentos de orden más técnico orientados hacia la industria. Por ejemplo, Morales-Vargas et al. (2020) y Rekik et al. (2018) presentan sendas revisiones con más de 700 y 500 de estos trabajos sobre calidad web, respectivamente.

Los primeros estudios surgieron de cara a evaluar la usabilidad o medida de eficacia, eficiencia y satisfacción (Nielsen, 2000) y, más recientemente, la experiencia de usuario (Garrett, 2011). Estas consideran la aplicación de testeos experimentales (Rubin y Chisnell, 2008) y métodos cuantitativos provenientes de la estadística (Sauro, 2010; Sauro y Lewis, 2012) y las métricas (Tullis y Albert, 2013). De igual modo, existe una segunda vertiente cuyos métodos se centran en el análisis de profesionales expertos. Sus reportes pueden tomar la forma de estándares (Bevan, 2005), reglas (Shneiderman, 2016), directrices (Leavitt y Shneiderman, 2006), principios (Tognazzini, 2014), heurísticas (Nielsen, 2020) o recomendaciones (Krug, 2014), entre otros.

Por otra parte, estas herramientas pueden ser de uso general, vale decir, que sirven para evaluar cualquier tipo de sitio (Codina, 2008); o corresponder a análisis sectoriales, que están especializados en un sector concreto. Tal es el caso del comercio electrónico, la educación, la sanidad, el gobierno, el turismo o los medios de comunicación (Pedraza-Jiménez et al., 2016).

Ejemplos de estos últimos instrumentos de evaluación de calidad web focalizados en cibermedios encontramos en trabajos pioneros como los de Abdullah y Wei (2008), basado en heurísticas de usabilidad; Zambarbieri et al. (2008), que aplica la técnica de eyetracking o seguimiento ocular; Rodríguez-Martínez et al. (2010), análisis experto destinado a analizar la adopción de la Web 2.0; Al-Radaideh et al. (2011), sustentado en el estudio de usuarios; Chung et al. (2012), sobre la base de encuestas; o el protocolo de análisis de contenidos propuesto por Martins (2012).

La presente investigación apunta precisamente a examinar este tipo de estudios. Busca, de manera general, caracterizar la literatura sobre la evaluación de la calidad web especializada en el sector mediático o aquel relativo a la prensa digital. Ello, con el objetivo de identificar sus diferentes metodologías e instrumentos y conocer sus principales autores. 
Y de forma particular, este trabajo pone especial atención en los atributos de calidad web (Hasan y Abuelrub, 2011; Olsina et al., 2006) que consideran estas herramientas y el grado de automatización con que se aplican. También si contemplan entre sus parámetros e indicadores específicos referencias a políticas internacionales de tecnología y comunicación, como los indicadores de universalidad de internet de la Unesco (2019), el reglamento general de protección de datos de la Unión Europea (2016) y otras directrices de calidad informativa.

Además, se plantea conocer si estos instrumentos toman en cuenta aspectos éticos y sociales donde la interfaz web tiene implicaciones, tales como: clickbait o titulares engañosos (García Orosa et al., 2017), privacidad de la información, rendición de cuentas (Mauri-Ríos y Ramon-Vegas, 2015), separación entre publicidad y contenido periodístico (Chung et al., 2012), venta de enlaces patrocinados (Lopezosa et al., 2019), declaración de fuentes de financiación, firma de los periodistas (The Trust Project, 2020), derechos de autor, y protección de la infancia (García de Torres y Farmer, 2017), entre otros.

Todos estos asuntos cobran gran relevancia en estos momentos en que existe una pérdida de credibilidad en los contenidos de internet debido a la proliferación de bulos, noticias falsas y desórdenes informativos (Guallar et al., 2020), acentuados en tiempos del coronavirus (Salaverría et al., 2020). Y en momentos en que la industria de los medios de comunicación pasa por una crisis de financiación en busca de nuevas fuentes de ingresos (Carvajal y Valero Pastor, 2018). Es en este contexto donde la evaluación de la calidad web en cibermedios surge como una disciplina que puede aportar un conjunto de herramientas de gran utilidad. Bajo esa hipótesis, este trabajo presenta una revisión y caracterización de los estudios existentes, los instrumentos que proponen y los principales indicadores que contemplan.

\section{Metodología}

Para el cumplimiento del objetivo descrito se utilizaron los principios de la revisión bibliográfica con aproximación sistemática (Booth et al., 2016). Específicamente para conformar el banco de documentos se empleó una revisión llevada a cabo con bases de datos académicas y un mapeo sistemático (Gough et al., 2017). Y como protocolo general para el análisis se aplicó el framework o marco de trabajo SALSA (Codina, 2018; Grant y Booth, 2009).

En primer lugar se definió la evaluación de calidad en sitios web de medios digitales como objeto de estudio. En cuanto al periodo de análisis, fue el comprendido entre 2000 y 2020. Como ecuación de búsqueda (Tabla 1) se escogieron los términos más comúnmente usados en la bibliografía, tanto en inglés como en castellano.

Estos se aplicaron sobre los campos de título, resumen y palabra clave de la colección principal de Web of Science (WoS) y Scopus, las bases de datos académicas multidisciplinarias más importantes. También se consultaron otras especializadas en el campo de la comunicación y las ciencias de la información, como la colección LISTA (Library, Information Science \& Technology Abstracts) de EBSCO Host o Emerald Insight, junto a los recursos de información Dialnet Plus, SciELO, Microsoft Academic y Dimensions. 
RLCS, Revista Latina de Comunicación Social, 80, 39-63

\begin{tabular}{ll|l|l|l} 
[Investigación] DOI: 10.4185/RLCS-2022-1515 & ISSN 1138-5820 & Año 2022
\end{tabular}

Tabla 1. Ecuaciones de búsqueda aplicadas en las bases de datos académicas.

\begin{tabular}{r}
\hline ["online news" OR "digital media" OR "online newspaper" OR "online media" OR "news website"] \\
AND [quality] AND \\
[assessment OR assess OR assessing OR assurance OR evaluation OR evaluating OR evaluate OR analysis \\
OR \\
index OR heuristic OR standard OR guideline] \\
ORestándar OR directriz] \\
AND [calidad] AND \\
[evaluación OR análisis OR "sistema de análisis" OR índice OR protocolo OR pauta OR guía OR heurística \\
["medio digital" OR cibermedio OR "diario electrónico" OR "periódico electrónico" OR "diario digital" OR \\
"periódico digital" OR "prensa digital" OR "noticia en línea"]
\end{tabular}

Por su amplia cobertura (Martín-Martín et al., 2018), y por incluir no solo artículos de revistas, sino también libros y otros documentos técnicos (Thelwall y Kousha, 2015) -altamente demandados en el ámbito profesional del desarrollo y diseño web-, se usó también el motor de búsqueda Google Académico. Desde allí se extrajeron además las citas recibidas por cada texto a agosto de 2021.

Como criterio de inclusión se consideró los documentos sobre calidad web en cibermedios publicados desde el año 2000. Se excluyeron, a su vez, los estudios basados solamente en estadísticas de visita o analítica web, documentos referidos únicamente a aplicaciones (Apps), e investigaciones centradas solo en los efectos o la psicología del usuario.

En la etapa de evaluación, los 532 trabajos recuperados se revisaron manualmente, examinando sus títulos y resúmenes, con el objetivo de verificar si se ajustaban con estos criterios. Con ello se redujo el alto ruido documental detectado y se descartaron falsos positivos. A continuación, en la fase de análisis se conformó un banco de 83 documentos, cuya información recogida se complementó con indicadores específicos sobre impacto y afiliación - esta última, recogida a partir de la unidad académica declarada por cada autor-, y se sistematizó en tres áreas referentes a datos de la publicación, los autores y el contenido (Tabla 2), a partir de la propuesta de análisis de la producción científica en medios digitales de Abadal y Guallar (2018).

Tabla 2. Resumen de las características observadas en las publicaciones.

\begin{tabular}{lcc}
\hline \multicolumn{1}{c}{ Publicación } & Autores & Contenido \\
\hline - Campos de información & - Ámbito de origen: académico, & - Factores de calidad web \\
bibliográfica: año, título, & profesional o normativo. & considerados. \\
formato, idioma, entre otras. & - Disciplina o área del & - Métodos y técnicas. \\
- Indicadores de impacto: citas & conocimiento y país, según la & - Instrumentos y herramientas. \\
recibidas en Google & afiliación declarada. & - Atributos de confianza. \\
Académico. & & \\
\hline
\end{tabular}

Con esa información, se realizó un análisis comparativo de las diferentes disciplinas o áreas del conocimiento que abordan la materia, a partir de dimensionar la presencia de las facultades o departamentos de comunicación. Entre los trabajos referidos a la calidad en el sector mediático se identificaron 22 textos planteados como propuesta de protocolo de evaluación general de cibermedios. En estos se analizó además su metodología, modo de aplicación y se sistematizaron los indicadores considerados como atributos de calidad web. Finalmente, se buscaron citas a estándares y políticas de tecnología y comunicación internacionales, así como la presencia o no en los criterios de análisis de aspectos de implicación ética, deontológica y social descritos en la introducción. 


\section{Resultados y discusión}

Entre los principales hallazgos se detecta que la producción científica sobre calidad web específica sobre el sector mediático o dedicado a la prensa digital presenta un moderado pero constante crecimiento las últimas dos décadas. Se observa una mayor concentración a partir del 2009 y destaca el año 2012 con diez publicaciones.

El total de trabajos pertenece al ámbito académico y, respecto al idioma de publicación, el banco de documentos lo conforman 50 en español, 31 en inglés y dos en portugués. En lo referente al formato, los artículos de revistas científicas son, con mucho, los que predominan, con la excepción de seis actas de congreso, cinco informes y cuatro tesis doctorales.

Según el análisis de la afiliación declarada, se identificó que un 53\% de los textos dedicados a cibermedios se generaron en universidades españolas. Siete se generaron en Estados Unidos, al que le siguen Reino Unido, Canadá, Brasil y Venezuela, con tres cada uno. También se hallaron trabajos de Austria, Ecuador, Italia, Malasia y otros ocho países.

A través de la sistematización de los nombres de escuelas, facultades o departamentos se pudo establecer que, del total de artículos revisados sobre calidad web en cibermedios, más de la mitad provienen del dominio de la comunicación (58\%). Le siguen los negocios -economía, administración, marketing y comercio- con un 15\%, las ciencias de la computación -informática y programación-con un 13\%, la biblioteconomía y documentación -ciencias de la información-con un $12 \%$, y un $2 \%$ que proviene de otras disciplinas.

Del total de trabajos analizados sobre calidad web en medios digitales, diez corresponden a artículos de revisión de aspectos teóricos o metodológicos sobre el análisis de cibermedios y nueve son estudios de caso aplicados a la prensa en internet. A su vez, 22 de los textos proponen un protocolo especializado en la evaluación de sitios web de medios digitales, con una metodología y un conjunto de indicadores explicados, con el fin de facilitar su aplicación. Finalmente, 40 trabajos presentan investigaciones centradas en el análisis de un parámetro en particular, como por ejemplo la participación, la usabilidad o el uso de multimedia.

\subsection{Estudios y casos}

La importancia de la interfaz web para los medios digitales es abordada en textos señeros sobre el estudio del periodismo digital (Salaverría, 2019). En su tipología de los cibermedios, Palacios y Díaz Noci (2009) proponen como primer punto de análisis el grado de desarrollo de los sitios y las acciones que pueden ejecutar los usuarios. Estas últimas son entendidas como el dinamismo o adaptación a la frecuencia de actualización, la interactividad (Cebrián-Herreros, 2009; Abadal y Guallar, 2018), la hipertextualidad y la multimedialidad (Masip et al., 2010).

De igual modo, Salaverría (2017) considera como primer factor tipológico al dispositivo con el cual se accede al cibermedio, y distingue aquellos formulados para la web, para tabletas, para móviles (Serm et al., 2006) y los multiplataforma o convergentes (Cabrera González, 2010). De ahí que otro factor a considerar previo al análisis es si el medio es "nativo digital" o proviene del proceso de convergencia desde otros soportes, impulsado por la evolución tecnológica.

Y una última dimensión a tener en cuenta a la hora de diseñar una evaluación de la calidad web de los cibermedios es su alcance. Entre los trabajos analizados encontramos tanto directrices para medios globales, como la BBC (Anderson y Egglestone, 2012); así como estudios de casos 
específicos sobre medios locales, como el diario VilaWeb, el primero de España sin referentes previos en papel (Iglesias-García y González-Díaz, 2012).

También investigaciones que comparan entre sí la prensa digital a escala continental, como en América Latina (Said-Hung y Arcila-Calderón, 2011b) o dentro de un mismo país, como es el caso de periódicos electrónicos iranís (Jowkar y Didegah, 2010), los portales de noticias malayos (Abdullah y Wei, 2008), o los cibermedios ecuatorianos (Odriozola Chéné et al., 2017), portugueses (Nunes et al., 2007) y venezolanos (Mogollón et al., 2006), entre otros. También los correspondientes a una región en particular, como los medios digitales de la Comunidad Valenciana en España (López García, 2008), o de los estados de São Paulo en Brasil (Rosa y Veras, 2013) o de Chihuahua en México (Salas Hernández et al., 2018).

\subsection{Protocolos de análisis}

Encontramos también trabajos que tienen por objetivo ofrecer modelos o sistemas para evaluar la calidad web de los medios digitales, con explicaciones metodológicas destinadas a que los puedan aplicar sus propios responsables u otros investigadores. La Tabla 3 presenta los protocolos de análisis para cibermedios con mayor cantidad de citas recibidas en Google Académico.

La lista la encabeza el trabajo de Rodríguez-Martínez et al. (2010), con un sistema de análisis con énfasis en la Web 2.0 y que es aplicado por otros seis estudios: Said-Hung y Arcila-Calderón (2011a, 2011b); Hernández Soriano y Cristóbal Fransi (2014a); Díaz-Campo (2014); Rodríguez-Martínez et al. (2012); y Salas Hernández et al. (2018).

A su vez, el protocolo de evaluación propuesto por Codina (2008) -cuya primera versión se remonta a 2000-, es aplicado, entre otros, en los estudios de Salaverría et al., (2004) sobre los periódicos vascos y navarros; o en el de Hernández Soriano y Cristóbal Fransi (2014b) en que también analizan VilaWeb. Incluso inspira o sirve de base para la generación de otros dos instrumentos específicos: el sistema de análisis de interactividad, buscabilidad y visibilidad de Linares, Codina, Vàllez, et al. (2016) y la escala de medición de la calidad percibida en cibermedios denominada e-SQ-Media (Hernández Soriano, 2015). Este último trabajo es el primero en hacer converger en un mismo modelo el estudio de los cibermedios y la calidad percibida, pues identifica los aspectos más exitosos en las diversas aplicaciones de ambos campos (Cristóbal Fransi et al., 2017).

Junto con el Sistema Articulado de Análisis de Cibermedios, SAAC (Codina et al., 2014), son las únicas herramientas diseñadas para evaluar integralmente la calidad de un medio digital, sin poner el foco en un parámetro en particular sobre otros, como en el resto de los trabajos. Difieren, eso sí, en el método empleado. Mientras e-SQ-Media se basa en la percepción de los usuarios, SAAC responde a la evaluación de profesionales.

\subsection{Métodos y herramientas}

La dicotomía entre los análisis expertos y los estudios de experiencia de usuario (UX) se evidencia en las investigaciones examinadas sobre calidad web en cibermedios. Al analizar sus métodos de aplicación (Gráfico 1), se detecta que más de la mitad corresponde al primer grupo y proponen inspecciones para ser aplicadas por investigadores especializados o profesionales con experiencia en el desarrollo de sitios web (Pribeanu, 2009; Usability.gov, 2013). 
Tabla 3. Protocolos de análisis de cibermedios con más citas en Google Académico (GA).

\begin{tabular}{|c|c|c|}
\hline Autores y año & Título & GA \\
\hline Rodríguez-Martínez et al. (2010) & $\begin{array}{l}\text { Cibermedios y web 2.0: modelo de análisis y resultados de } \\
\text { aplicación }\end{array}$ & 193 \\
\hline Rodríguez-Martínez et al. (2012) & $\begin{array}{l}\text { Indicadores para la evaluación de la calidad en } \\
\text { cibermedios: análisis de la interacción y de la adopción de } \\
\text { la Web } 2.0\end{array}$ & 139 \\
\hline Fondevila Gascón (2014) & $\begin{array}{l}\text { El uso de hipertexto, multimedia e interactividad en } \\
\text { periodismo digital: propuesta metodológica de ranking de } \\
\text { calidad }\end{array}$ & 76 \\
\hline Romero-Rodríguez et al. (2016) & $\begin{array}{l}\text { Dimensiones e indicadores de la calidad informativa en los } \\
\text { medios digitales }\end{array}$ & 54 \\
\hline Aranyi y van Schaik (2016) & Testing a model of user-experience with news websites & 52 \\
\hline Codina et al. (2014) & $\begin{array}{l}\text { Sistema Articulado de Análisis de Cibermedios (SAAC): } \\
\text { Una propuesta sobre el qué y el cómo para estudiar medios } \\
\text { de comunicación digitales }\end{array}$ & 44 \\
\hline $\begin{array}{l}\text { García-Carretero, Codina, Díaz-Noci, } \\
\text { et al. (2016) }\end{array}$ & $\begin{array}{l}\text { Herramientas e indicadores SEO: características y } \\
\text { aplicación para análisis de cibermedios }\end{array}$ & 33 \\
\hline $\begin{array}{l}\text { Aguirre Mayorga y Bernal Suárez, } \\
\text { (2014) }\end{array}$ & $\begin{array}{l}\text { Contenidos periodísticos digitales: hacia un modelo de } \\
\text { medición de calidad }\end{array}$ & 26 \\
\hline Sánchez-González y Alonso (2012) & $\begin{array}{l}\text { Propuesta metodológica para el análisis de las tecnologías } \\
\text { de participación en cibermedios }\end{array}$ & 26 \\
\hline Al-Radaideh et al., (2011) & $\begin{array}{l}\text { Usability evaluation of online news websites: a user } \\
\text { perspective approach }\end{array}$ & 24 \\
\hline Linares et al. (2016) & $\begin{array}{l}\text { Interactividad, buscabilidad y visibilidad en cibermedios: } \\
\text { sistema de análisis y resultados }\end{array}$ & 15 \\
\hline Cristóbal Fransi et al. (2017) & $\begin{array}{l}\text { Critical factors in the evaluation of online media: creation } \\
\text { and implementation of a measurement scale (e-SQ-Media) }\end{array}$ & 11 \\
\hline Limia Fernández et al. (2013) & $\begin{array}{l}\text { Interactividad y participación en los cibermedios: una } \\
\text { propuesta metodológica para la elaboración, registro y } \\
\text { análisis de datos }\end{array}$ & 11 \\
\hline Linares et al. (2015) & $\begin{array}{l}\text { Interactividad en cibermedios: Propuesta nuclear de } \\
\text { protocolo de análisis }\end{array}$ & 11 \\
\hline $\begin{array}{l}\text { García-Carretero, Codina y Pedraza- } \\
\text { Jiménez (2016) }\end{array}$ & $\begin{array}{l}\text { Indicadores para el estudio de la visibilidad y del impacto } \\
\text { de los cibermedios en el ecosistema digital: mapeo y } \\
\text { caracterización de herramientas de análisis SEO online }\end{array}$ & 10 \\
\hline Sanabre Vives (2015) & $\begin{array}{l}\text { Un modelo para el análisis y concepción de sitios web: El } \\
\text { WebSite Canvas Model aplicado a Eldiario.es }\end{array}$ & 6 \\
\hline Hernández Soriano (2015) & $\begin{array}{l}\text { E-SQ-Media, creación e implementación de una escala de } \\
\text { medición de la calidad percibida en cibermedios }\end{array}$ & 4 \\
\hline
\end{tabular}




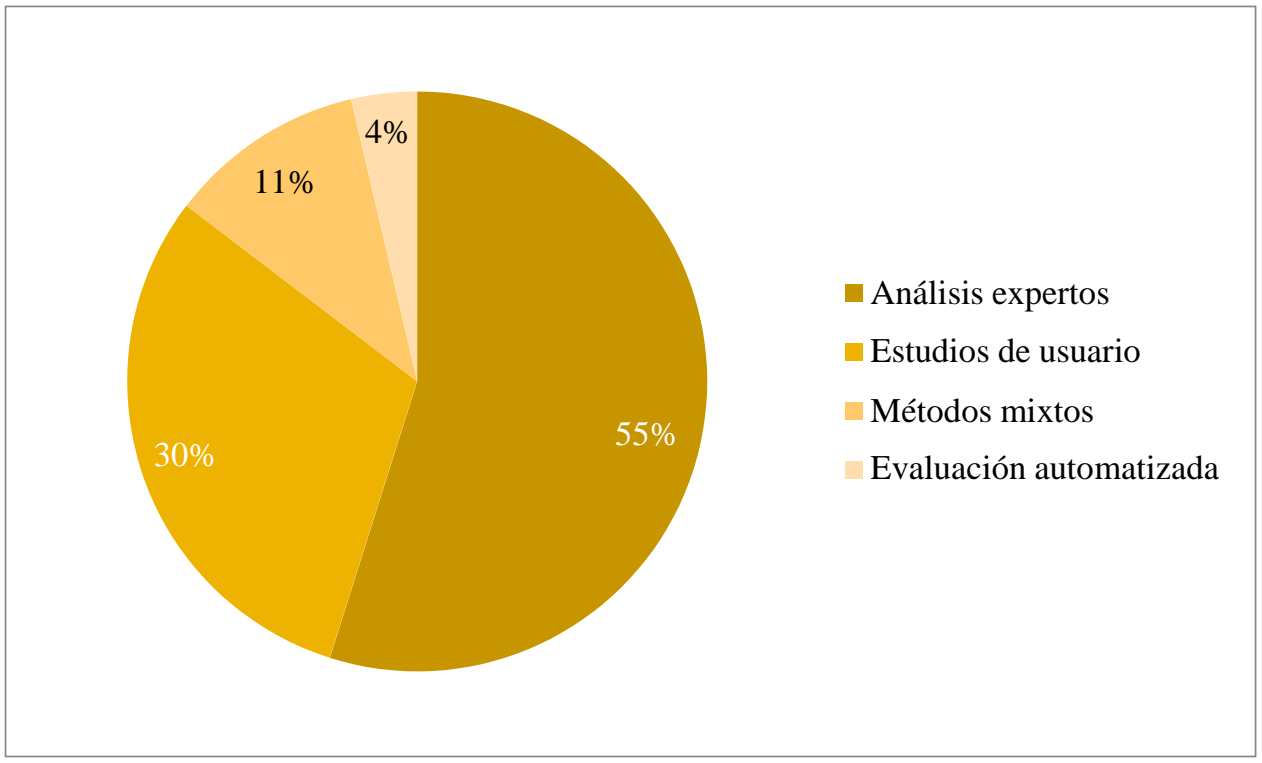

Gráfico 1. Métodos de evaluación de calidad web presentes en los trabajos analizados.

Entre las técnicas y herramientas más utilizadas (Tabla 4) en este método encontramos la evaluación heurística, el análisis de contenido, las listas o preguntas de chequeo, los índices y los sistemas articulados, entre otros. Por su parte, los estudios de usuarios detectados se sustentan principalmente en encuestas, cuestionarios de satisfacción, pruebas de usabilidad, experimentos, entrevistas, test $\mathrm{A} / \mathrm{B}$, seguimiento de tareas y técnicas como el think aloud en que las personas van "pensando en voz alta" y narrando sus impresiones mientras navegan un sitio web y utilizan sus funciones.

Es frecuente que también se empleen técnicas mixtas que combinan pruebas de usabilidad con estudios de percepción. En estos se aplican cuestionarios donde los usuarios responden posteriormente sobre su valoración acerca de la eficacia, eficiencia y satisfacción frente a los medios de comunicación en internet, para luego ponderarlos mediante modelos matemáticos con múltiples factores, como el formulado por Cristóbal-Fransi et al. (2017).

Solamente cuatro de los estudios están basados en experimentación directa con usuarios: Zambarbieri et al. (2008) utilizan el método de rastreo ocular en la lectura (eye tracking) aplicado a periódicos italianos; Aranyi et al., (2012) basan su trabajo en pruebas psicométricas en sitios de noticias; mientras que $\mathrm{Yu}$ y Kong (2016) realizan test A/B sobre navegación web en pantallas pequeñas.

Por su parte, O’Brien y Lebow (2013), proponen una combinación de técnicas de observación y de respuesta a estímulos. Entre los métodos considerados incluyeron escalas psicométricas participación del usuario, absorción cognitiva, escalas de usabilidad del sistema-, el interés autoinformado en el contenido de las noticias y métricas de rendimiento -tiempo de lectura, tiempo de navegación, número de páginas visitadas y uso de los enlaces recomendados-; y las reacciones fisiológicas -frecuencia cardíaca, actividad electrodérmica y electrocitograma- registradas durante la interacción.

También cabe mencionar la presencia de tres trabajos sobre inspecciones automatizadas mediante métodos computacionales o de inteligencia artificial. Di Massa et al. (2010) utilizan el procesamiento del lenguaje natural para generar un sistema de recomendación de noticias basado en modelos de interés de los usuarios y análisis de contenido multimodal. 
RLCS, Revista Latina de Comunicación Social, 80, 39-63

[Investigación] DOI: 10.4185/RLCS-2022-1515| ISSN 1138-5820| Año 2022

Tabla 4. Herramientas metodológicas presentes en los trabajos de calidad web en cibermedios

\begin{tabular}{|c|c|c|c|}
\hline \multicolumn{2}{|l|}{ Técnicas } & \multicolumn{2}{|l|}{ Instrumentos } \\
\hline Evaluación heurística & 27 & Preguntas de chequeo & 9 \\
\hline Encuesta & 11 & Cuestionario de satisfacción & 5 \\
\hline Pruebas de usabilidad & 7 & Escala de puntuación & 4 \\
\hline Análisis de contenido & 5 & Índice & 4 \\
\hline Entrevista & 5 & Escala psicométrica & 3 \\
\hline Think aloud & 3 & Test A/B & 2 \\
\hline Procesamiento del lenguaje natural (PLN) & 2 & Baremos & 1 \\
\hline Análisis de correspondencias & 1 & Eyectracking & 1 \\
\hline Aprendizaje automático supervisado & 1 & Heurístico & 1 \\
\hline Aprendizaje profundo (deep learning) & 1 & Índices SEO & 1 \\
\hline Design thinking & 1 & Conteo de clics & 1 \\
\hline Diseño centrado en el usuario & 1 & Tiempo por tarea & 1 \\
\hline Estímulos experimentales & 1 & & \\
\hline Técnica mixta & 1 & & \\
\hline Modelos & & Software & \\
\hline Sistemas articulados & 11 & Alexa & 14 \\
\hline SAAC & 6 & Moz & 4 \\
\hline e-SQ-Media & 3 & Majestic & 3 \\
\hline WCAG 2.0 & 3 & Ahrefs & 2 \\
\hline eWOM & 1 & SEMrush & 2 \\
\hline \multirow[t]{4}{*}{ WebSite Canvas Model } & 1 & eXaminator & 1 \\
\hline & & Similar Web & 1 \\
\hline & & Sistrix & 1 \\
\hline & & WebXACT & 1 \\
\hline
\end{tabular}

Asimismo, Omidvar et al. (2020) usan el deep learning o aprendizaje profundo para determinar la calidad de los titulares de las noticias publicadas mediante el recuento de clics y el tiempo de permanencia de los visitantes, con el objetivo de predecir los titulares que mejor invitan a la lectura. Por otro lado, Scharkow (2013) analiza el contenido de los cibermedios alemanes a través de técnicas de machine learning, concretamente algoritmos de aprendizaje automático supervisado.

Finalmente, destaca la utilización de instrumentos para medir atributos específicos, como son los casos de las guías WCAG 2.0 o WebXACT para evaluar la accesibilidad web. También de softwares especializados, como Alexa y Similar Web para medir el tráfico web, o Ahrefs, Majestic SEO, Moz, SEMrush o Sistrix, para dimensionar la visibilidad y el posicionamiento web de los cibermedios (García-Carretero, Codina, y Pedraza-Jiménez, 2016).

\subsection{Parámetros e indicadores}

Un factor en común en casi todas las publicaciones que plantean protocolos de evaluación de la calidad web en medios digitales es que se organizan en parámetros (Tabla 5) e indicadores. Los primeros son características conceptuales y responden a la pregunta sobre qué queremos estudiar. Los segundos, a cómo vamos a hacer el estudio. Son los elementos de análisis y permiten "operacionalizar" los parámetros. Su presencia puede ser verificada a través de una inspección mediante listas o preguntas de chequeo (Codina et al., 2014). 
RLCS, Revista Latina de Comunicación Social, 80, 39-63

[Investigación] DOI: 10.4185/RLCS-2022-1515| ISSN 1138-5820| Año 2022

Tabla 5. Parámetros de calidad web en los protocolos de evaluación de cibermedios más citados

\begin{tabular}{|c|c|}
\hline Trabajo & Parámetros \\
\hline $\begin{array}{l}\text { Rodríguez-Martínez et al. } \\
\text { (2010) }\end{array}$ & $\begin{array}{l}\text { Accesibilidad; Visibilidad y popularidad; Acceso a la información; } \\
\text { Profundización en la información; Herramientas de interacción; } \\
\text { Personalización de la información; Herramientas Web 2.0; Difusión en redes } \\
\text { sociales }\end{array}$ \\
\hline $\begin{array}{l}\text { Rodríguez-Martínez et al. } \\
\text { (2012) }\end{array}$ & $\begin{array}{l}\text { Interacción; Participación; Acceso al contenido; Personalización; Versiones; } \\
\text { Herramientas Web 2.0; Plataformas Web } 2.0\end{array}$ \\
\hline Fondevila Gascón (2014) & $\begin{array}{l}\text { Hipertextualidad; Texto; Fotografía; Vídeo; Audio; Infografía; Comentarios en } \\
\text { el foro; Entrevistas online }\end{array}$ \\
\hline $\begin{array}{l}\text { Romero-Rodríguez et al. } \\
\text { (2016) }\end{array}$ & $\begin{array}{l}\text { Ámbitos empresariales; Ámbitos socio-laborales de los trabajadores; Ámbitos } \\
\text { de contenido informativo y producto final }\end{array}$ \\
\hline Aranyi y van Schaik (2016) & Impresión; Contenido; Diseño; Arquitectura de la información; Distracción \\
\hline $\begin{array}{l}\text { García-Carretero, Codina, } \\
\text { Díaz-Noci et al. (2016) }\end{array}$ & $\begin{array}{l}\text { Authority (Moz); Trust Flow (Majestic); Citation Flow (Majestic); Global } \\
\text { Rank (Ahrefs); URL Rating (Ahrefs); Domain Rating (Ahrefs); Global Rank } \\
\text { (Alexa); Local Rank (Alexa) }\end{array}$ \\
\hline $\begin{array}{l}\text { Aguirre Mayorga y Bernal } \\
\text { Suárez (2014) }\end{array}$ & $\begin{array}{l}\text { Agenda informativa; Profundidad en el manejo de la información y } \\
\text { documentación; Diversificación en el uso de géneros periodísticos; } \\
\text { Multimedialidad; Interactividad; Hipertextualidad }\end{array}$ \\
\hline Al-Radaideh et al., (2011) & Usabilidad; Contenido; Diseño web \\
\hline $\begin{array}{l}\text { Limia Fernández et al. } \\
\text { (2013) }\end{array}$ & Personalización; Web 1.0; Web 2.0; Redes sociales \\
\hline Linares et al. (2015) & $\begin{array}{l}\text { Relación cibermedio-usuario; Contenidos generados por usuarios; } \\
\text { Buscabilidad y Navegación }\end{array}$ \\
\hline $\begin{array}{l}\text { Hernández Soriano (2015); } \\
\text { Cristóbal Fransi et al. } \\
\text { (2017) }\end{array}$ & $\begin{array}{l}\text { Eficiencia; Disponibilidad del sistema; Confiabilidad y privacidad; } \\
\text { Interacción; Calidad percibida; Satisfacción del usuario; Lealtad del usuario }\end{array}$ \\
\hline Sanabre Vives (2015) & $\begin{array}{l}\text { Socios tecnológicos; Socios de contenido; Actividades clave; Recursos clave; } \\
\text { Propuesta de valor; Segmentos de clientes; Relación con los clientes; Canal; } \\
\text { Flujo de ingresos; Estructura de costes; Benchmarking; Debilidades de la } \\
\text { competencia; Social, local y móvil }\end{array}$ \\
\hline
\end{tabular}

Entre más de 170 parámetros e indicadores distintos presentes en los trabajos examinados (Gráfico 2), hay algunos que concitan una atención especial entre los autores, quienes formulan protocolos específicos para su análisis. El más estudiado es el de la interactividad e interacción (CebriánHerreros, 2009; Linares et al., 2015) como factor determinante en la calidad de los medios digitales (Hernández Soriano y Cristóbal Fransi, 2016). Además del muy citado sistema para medir la adopción de la Web 2.0 (Rodríguez-Martínez et al., 2012), Linares et al. (2015) proponen otro basado en la relación cibermedio-usuario, los contenidos generados por estos últimos, y la buscabilidad y la navegación.

Muy ligado al anterior está el indicador de la participación (Eberwein, 2019; Ksiazek, 2018), donde también encontramos propuestas destinadas a su estudio en particular: Sánchez-González y Alonso (2012) se centran en el análisis de las tecnologías, mientras que Limia Fernández et al. (2013) desarrollan un método para la elaboración, registro y análisis de datos sobre los procesos de interacción y participación en medios digitales. 
RLCS, Revista Latina de Comunicación Social, 80, 39-63

[Investigación] DOI: 10.4185/RLCS-2022-1515| ISSN 1138-5820|Año 2022

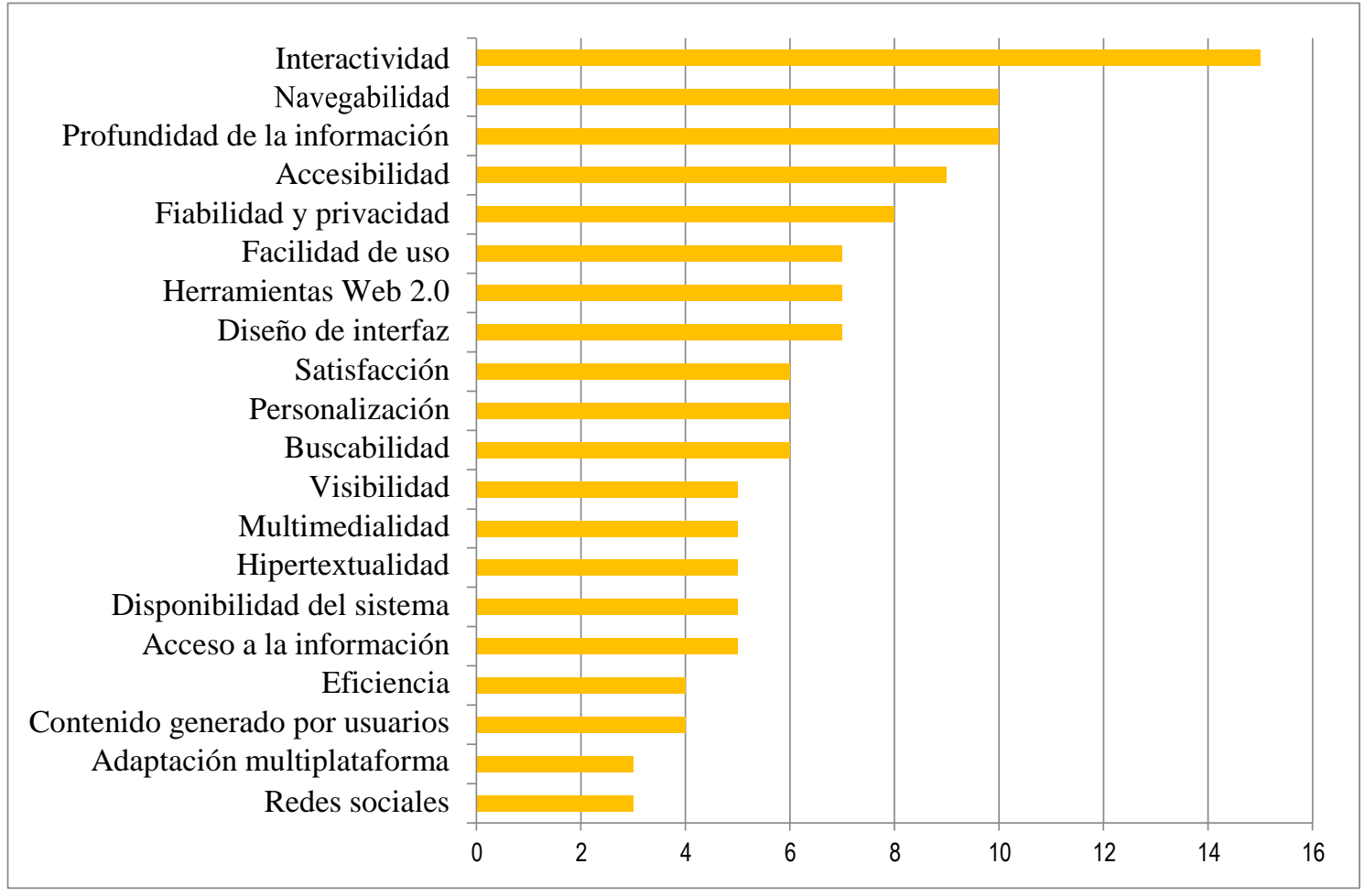

Gráfico 2. Indicadores de calidad web en cibermedios con más menciones en los trabajos analizados

La usabilidad (Bevan, 2005; ISO, 2018; Nielsen, 2000) como medida de eficacia, eficiencia y satisfacción de los usuarios también es ampliamente abordada, tanto en sitios web de periódicos digitales (Al-Radaideh et al., 2011; Mariage y Vanderdonckt, 2001; Rosa y Veras, 2013) como en dispositivos móviles (Jeong y Jung Han, 2012; Jiménez Iglesias, 2018). Lo propio ocurre con el acceso universal o accesibilidad (Salas Hernández et al., 2018) y la personalización.

De igual modo, la experiencia de usuario (Garrett, 2011) cuenta con trabajos específicos como los de Flavián y Gurrea (2006a, 2006b, 2007), quienes desarrollan una línea de investigación en torno a influencia de los atributos de calidad web en los lectores a la hora de elegir un diario en internet. Rabaya Toma et al. (2018), a su vez, exploran la satisfacción en la lectura y su impacto en la posterior intención de los usuarios en volver a visitar el sitio o recomendarlo.

Otro parámetro analizado es la arquitectura de información (Rosenfeld et al., 2015), con gran número de menciones a indicadores como la navegación, la organización de los contenidos y el acceso a la información, así como el propio diseño gráfico de la interfaz. No obstante, no se detectan protocolos específicos para su análisis.

Y, por supuesto, el contenido también ocupa un lugar destacado, con la profundidad de las informaciones, la fiabilidad y la credibilidad -detalladas en el apartado siguiente-. También con atributos propios de los medios digitales, como la multimedialidad (Guallar et al., 2010) y la hipertextualidad (Fondevila Gascón, 2014; Masip et al., 2010), incluyendo indicadores especiales como la infografía interactiva (Langer y Zeiller, 2017; Túñez López y Nogueira, 2017), la transmisión de video en directo a través de plataformas sociales (Apablaza-Campos et al., 2020; Apablaza-Campos y Codina, 2018) y el análisis de la temporalidad (Freixa, 2020).

Por otra parte, cabe mencionar además los indicadores de impacto, como la experiencia de búsqueda (Lopezosa, Iglesias-García, et al., 2020); el posicionamiento web (Lopezosa, Codina, et al., 2020; 
Lopezosa y Codina, 2018); el tráfico (Jowkar y Didegah, 2010); y la visibilidad en internet (GarcíaCarretero, Codina, y Pedraza-Jiménez, 2016; García-Carretero, Codina, Díaz-Noci, et al., 2016; Linares, Codina, Vàllez, et al., 2016), donde los autores realizan un mapeo y caracterización de herramientas e indicadores de optimización para buscadores o SEO -search engine optimization-. En la misma línea, Lopezosa, García-Carretero, et al. (2020) presentan el sistema de análisis de la visibilidad en cibermedios denominado AVCIS, al tiempo que García-Carretero et al. (2020) plantean una metodología especializada para medios nativos digitales.

El factor estratégico o de rendimiento frente a la audiencia es abordado también por Sanabre Vives (2015) en el WebSite Canvas Model con que evalúa el medio español Eldiario.es como estudio de caso. Finalmente, es único el framework o marco de trabajo propuesto por Serm et al. (2006), que de forma pionera, aborda las tareas para desarrollar y evaluar cibermedios en dispositivos móviles.

\subsection{Atributos de confianza}

Por otra parte, son menos numerosas las referencias detectadas a atributos de confianza o asuntos de implicación ética y social. Ello a pesar de que en la credibilidad de las noticias influyen tanto factores tradicionales del rigor periodístico como aspectos tecnológicos, en los cuales el sitio web del medio es determinante.

En concreto, en su investigación Chung et al. (2012) demuestran empíricamente las relaciones entre las características tecnológicas de los cibermedios -como la hipertextualidad de fuentes diversas en las noticias en línea- y las posteriores evaluaciones de credibilidad. A su vez, Martín-Sanromán et al. (2019) señalan, por ejemplo, que prácticas como los enlaces engañosos -clicbait- o la publicidad invasiva en un diario online hacen pensar que son diseños orientados más al anunciante o al propio medio, que al lector.

Entre las menciones encontradas, Rodríguez-Martínez et al. (2012) identifican como un valor si se menciona al periodista autor de la noticia y si existe algún correo electrónico de contacto con este o con la redacción. Del mismo modo, señalan como algo positivo si usuario puede sugerir correcciones o modificaciones en el contenido publicado por el medio.

En la misma línea, Salaverría et al. (2004) valoran también la opción de reportar errores en la información por parte de los lectores. Al igual que Linares et al. (2016), quienes añaden el atributo de la confidencialidad, entendida como que el medio de comunicación permita al usuario compartir de forma anónima y segura materiales y mensajes.

A su vez, la protección de datos personales es señalada por Hernández Soriano y Cristóbal Fransi (2014) como un factor para construir confianza en los lectores. Esta también es reforzada con la ética periodística y el estilo de escritura (Hope y Li, 2004), así como con el manejo de la información y la documentación en profundidad (Aguirre Mayorga y Bernal Suárez, 2014).

El estudio que menciona explícitamente estos aspectos éticos es el de Romero-Rodríguez et al., (2016), que alude a la conveniencia de la presencia de códigos y normativas éticas del propio medio. De igual modo, en su protocolo proponen como atributo consignar la información de transparencia y los vínculos e intereses económicos de la empresa. En la misma línea, Martins (2012) plantea un protocolo de calidad para contenidos en el periodismo digital, basado en la medición de la presencia, carga e intensidad de un conjunto de valores en los periódicos digitales. 
En los instrumentos de evaluación de calidad web en cibermedios analizados no se detectaron parámetros e indicadores específicos para analizar la veracidad de los titulares o la debida separación entre la publicidad y el contenido periodístico. Tampoco sobre la presencia de espacios para la declaración de intereses, la política de privacidad y protección de datos, la rendición de cuentas o la defensa del lector, entre otros.

De igual modo, no se hallaron referencias a políticas internacionales de tecnología y comunicación, como las de Unesco o la UE, o a proyectos periodísticos internacionales para la evaluación de la credibilidad en las noticias, como el protocolo The Trust Indicators and their Attributes (The Trust Project, 2020). Solamente menciones a estándares técnicos como las guías de accesibilidad del World Wide Web Consortium (W3C).

\section{Conclusiones}

Coincidentemente con los trabajos de Rekik et al. (2018) y Morales-Vargas et al. (2020), se detecta que los estudios sobre la calidad web en el sector mediático presentan una tendencia creciente durante los últimos veinte años. En especial en la disciplina de la comunicación están despertando interés por su relación directa con la sostenibilidad de los medios, aunque comparten espacio con otras áreas del conocimiento Ugras et al. (2016).

En esta investigación se han analizado más de ochenta trabajos y detectado una veintena que proponen protocolos específicos para examinar la calidad de los sitios web de los medios digitales. Las metodologías más utilizadas en ellos son el análisis experto mediante evaluación heurística y listas de chequeo - con algunos autores que hacen énfasis en la necesidad de que estas listas no se limiten solo preguntas sino que incorporen procedimientos explícitos de evaluación experta-. Por el contrario, los estudios de usuario y los procedimientos computacionales o automatizados son mucho menos frecuentes. Esto contrasta con otros sectores (Allison et al., 2019) o el ámbito profesional (Rosala y Krause, 2020), donde hay mayor presencia de métodos participativos y experimentales.

Como también señalan Abadal y Guallar (2018), el parámetro más estudiado es la interactividad, especialmente a través de herramientas de la web social y a partir del contenido generado por el usuario. Le sigue la usabilidad y la experiencia de usuario, con indicadores como accesibilidad, personalización, facilidad de uso, eficacia, eficiencia y satisfacción.

La arquitectura de información también es objeto de análisis, con la navegabilidad, la organización y el acceso a la información, así como el diseño de interfaz, entre muchos otros atributos. Al respecto no se detectan instrumentos específicos para su estudio, como sí existen para el análisis de factores estratégicos y de la visibilidad o el posicionamiento en buscadores.

Y desde luego, el contenido también ocupa un lugar destacado. Para su evaluación, los instrumentos especializados consideran el análisis de indicadores tales como la profundidad de las informaciones, la fiabilidad, la multimedialidad y la hipertextualidad.

Sin embargo, entre estos se hallan muy pocas referencias a atributos de confianza, rigor periodístico o asuntos éticos o deontológicos, y en ningún caso se citan políticas de comunicación y tecnología internacionales. Este hecho llama la atención especialmente si se toman en cuenta los últimos años, debido a fenómenos recientes como la desinformación y el que la industria de los medios, y en particular la prensa, afronte una crisis de financiación y credibilidad a escala global. 
Esta relativa carencia señala claramente un hueco de investigación. Esta es una de las funciones de las revisiones bibliográficas sistematizadas como herramienta metodológica -como la aplicada en este trabajo-, además de presentar los rasgos más significativos del tema estudiado.

En todo caso, la calidad de sitios web de los medios digitales y sus instrumentos de análisis sectoriales son claramente un campo de estudio emergente, que se vuelve cada vez más especializado y diverso. Una razón probable son los nuevos modelos de negocio de los medios de comunicación, una vez constatada la baja continua en la venta de ejemplares impresos y el descenso de los ingresos publicitarios. Estas dos tendencias hacen cada vez más importantes los estudios sobre el propio soporte digital de los cibermedios.

De este modo, en esta investigación además de proporcionar una visión sobre los aspectos más significativos de la calidad web aplicada a medios de comunicación -que puede ser útil a otros investigadores y a los profesionales de la prensa-, se ha detectado que en el ámbito de la evaluación de cibermedios existe una interesante y amplia oportunidad de trabajos futuros. Más aún en estos tiempos de fake news, donde se refuerza la necesidad de desarrollar instrumentos integrales que no solo consideren indicadores técnicos y estratégicos, sino también de atributos de confianza

\section{Referencias}

Abadal, E., \& Guallar, J. (2018). Scientific production on interaction in digital news media. En M. Pérez-Montoro (Ed.), Interaction in Digital News Media: From Principles to Practice (pp. 175191). Palgrave Macmillan. https://doi.org/10.1007/978-3-319-96253-5_8

Abdullah, R., \& Wei, K. T. (2008). Usability Measurement of Malaysia Online News Websites. IJCSNS International Journal of Computer Science and Network Security, 8(5).

Aguirre Mayorga, C., \& Bernal Suárez, J. D. (2014). Contenidos periodísticos digitales: hacia un modelo de medición de calidad. ComHumanitas, 5(1), 75-90.

Al-Radaideh, Q. A., Abu-Shanab, E., Hamam, S., \& Abu-Salem, H. (2011). Usability evaluation of online news websites: A user perspective approach. World Academy of Science, Engineering and Technology, 50, 1049-1057.

Allison, R., Hayes, C., McNulty, C. A. M., \& Young, V. (2019). A Comprehensive Framework to Evaluate Websites: Literature Review and Development of GoodWeb. JMIR formative research, 3(4), e14372. https://doi.org/10.2196/14372

Anderson, P. J., \& Egglestone, P. (2012). The development of effective quality measures relevant to the future practice of BBC news journalism online. Journalism: Theory, Practice \& Criticism, 13(7), 923-941. https://doi.org/10.1177/1464884912457533

Apablaza-Campos, A., \& Codina, L. (2018). Social Media Live Streaming: estudio de caso y diseño de matriz de análisis. Cuadernos.info, 43, 161-179. https://doi.org/10.7764/cdi.43.1342

Apablaza-Campos, A., Morales-Vargas, A., Lopezosa, C., Salvat, J., \& Codina, L. (2020). Social Media Live Streaming (SMLS) en medios digitales: el uso de Periscope, Facebook Live y YouTube Live en cibermedios chilenos. Dígitos. Revista de Comunicación Digital, 6, 219-238. https://doi.org/10.7203/RD.V1I6.145 
Aranyi, G., \& van Schaik, P. (2016). Testing a model of user-experience with news websites. Journal of the Association for Information Science and Technology, 67(7), 1555-1575. https://doi.org/10.1002/asi.23462

Aranyi, G., van Schaik, P., \& Barker, P. (2012). Using think-aloud and psychometrics to explore users' experience with a news Web site. Interacting with Computers, 24(2), 69-77. https://doi.org/10.1016/j.intcom.2012.01.001

Bevan, N. (2005). Guidelines and standards for web usability. Proceedings of the 11th International Conference on Human-Computer Interaction, Las Vegas (HCI International), 22-27.

Booth, A., Sutton, A., \& Papaioannou, D. (2016). Systematic approaches to a successful literature review (2nd ed.). SAGE Publications Ltd. https://uk.sagepub.com/en-gb/eur/systematicapproaches-to-a-successful-literature-review/book244586

Cabrera González, M. Á. (2010). Evolución tecnológica y cibermedios. Comunicación Social.

Carvajal, M., \& Valero Pastor, J. M. (2018). Revenue streams within Spain's journalism industry, according to its editors. Hipertext.net: Revista Académica sobre Documentación Digital y Comunicación Interactiva, O(17), 83-94. https://doi.org/10.31009/hipertext.net.2018.i17.08

Cebrián-Herreros, M. (2009). Comunicación interactiva en los cibermedios. Comunicar, 17(33), 1524. https://doi.org/10.3916/c33-2009-02-001

Chung, C. J., Nam, Y., \& Stefanone, M. A. (2012). Exploring online news credibility: The relative influence of traditional and technological factors. Journal of Computer-Mediated Communication, 17(2), 171-186. https://doi.org/10.1111/j.1083-6101.2011.01565.x

Codina, L. (2008). Sistema general de análisis y evaluación de sitios web: parámetros e indicadores. En Metodología de análisis y evaluación de recursos digitales en línea (Vol. 1). http://www.lluiscodina.com/wp-content/uploads/2014/04/indicadores_2008.pdf

Codina, L. (2018). Revisiones bibliográficas sistematizadas: procedimientos generales y framework para ciencias humanas y sociales. Departamento de Comunicación, Universitat Pompeu Fabra. https://repositori.upf.edu/handle/10230/34497

Codina, L., Pedraza-Jiménez, R., Díaz-Noci, J., Rodríguez-Martínez, R., Pérez-Montoro, M., \& Cavaller-Reyes, V. (2014). Sistema Articulado de Análisis de Cibermedios (SAAC): Una propuesta sobre el qué y el cómo para estudiar medios de comunicación digitales. Hipertext.net: Revista Académica sobre Documentación Digital y Comunicación Interactiva, O(12). http://raco.cat/index.php/Hipertext/article/view/275560

Cristóbal Fransi, E., Hernández Soriano, F., \& Marimon, F. (2017). Critical factors in the evaluation of online media: creation and implementation of a measurement scale (e-SQ-Media). Universal Access in the Information Society, 16(1), 235-246. https://doi.org/10.1007/s10209-016-0454-3

Di Massa, R., Montagnuolo, M., \& Messina, A. (2010). Implicit news recommendation based on user interest models and multimodal content analysis. Proceedings of the $3 r d$ international workshop on Automated information extraction in media production - AIEMPro '10, 33. https://doi.org/10.1145/1877850.1877861 
Díaz-Campo, J. (2014). Las cadenas de televisión españolas en Internet: un estudio sobre la calidad de sus sitios web. Estudios sobre el Mensaje Periodístico, 20(1). https://doi.org/10.5209/rev_ESMP.2014.v20.n1.45219

Díaz Noci, J., \& Salaverría, R. (2003). Manual de redacción ciberperiodística. Editorial Ariel.

Eberwein, T. (2019). «Trolls» or «warriors of faith»? Differentiating dysfunctional forms of media criticism in online comments. Journal of Information, Communication and Ethics in Society, ahead-of-p(ahead-of-print), 131-143. https://doi.org/10.1108/JICES-08-2019-0090

Flavián, C., \& Gurrea, R. (2006a). The choice of digital newspapers: influence of reader goals and user experience. Internet Research, 16(3), 231-247. https://doi.org/10.1108/10662240610673673

Flavián, C., \& Gurrea, R. (2006b). The role of readers' motivations in the choice of digital versus traditional newspapers. Journal of Targeting, Measurement and Analysis for Marketing, 14(4), 325-335. https://doi.org/10.1057/palgrave.jt.5740192

Flavián, C., \& Gurrea, R. (2007). Analyzing the influence of websites attributes on the choice of newspapers on the internet. En E-Commerce and Web Technologies (pp. 179-190). Springer Berlin Heidelberg. https://doi.org/10.1007/978-3-540-74563-1_18

Fondevila Gascón, J. F. (2014). El uso de hipertexto, multimedia e interactividad en periodismo digital: propuesta metodológica de ranking de calidad. Zer, 19(36), 55-76. https://addi.ehu.es/handle/10810/41163

Freixa, P. (2020). Herramientas e indicadores para el análisis de la temporalidad en el periodismo digital y el documental interactivo. Comunicación y diversidad. Selección de comunicaciones del VII Congreso Internacional de la Asociación Española de Investigación de la Comunicación (AEIC), 25-37. https://doi.org/10.3145/AE-IC-epi.2020.e02

García-Carretero, L., Codina, L., Díaz-Noci, J., \& Iglesias-García, M. (2016). Herramientas e indicadores SEO: características y aplicación para análisis de cibermedios. El Profesional de la Información, 25(3), 497. https://doi.org/10.3145/epi.2016.may.19

García-Carretero, L., Codina, L., \& Pedraza-Jiménez, R. (2016). Indicadores para el estudio de la visibilidad y del impacto de los cibermedios en el ecosistema digital: mapeo y caracterización de herramientas de análisis SEO online. http://hdl.handle.net/10230/27455

García-Carretero, L., Lopezosa, C., \& Codina, L. (2020). Análisis de visibilidad de los medios nativos digitales en Internet: Propuesta metodológica y resultados. En K. Meso Ayerdi, S. Peña Fernández, \& A. Larrondo Ureta (Eds.), XI Congreso Internacional de Ciberperiodismo. Nuevos actores y estrategias en el espacio mediático compartido (pp. 115-145). Universidad del País Vasco. https://dialnet.unirioja.es/servlet/articulo?codigo $=7433488$

García de Torres, E., \& Farmer, Y. (2017). Ética en los medios de comunicación: retos y oportunidades para la investigación. Profesional de la Información, 26(2), 153-157. https://doi.org/10.3145/epi.2017.mar.01 
García Orosa, B., Gallur Santorun, S., \& López García, X. (2017). El uso del clickbait en cibermedios de los 28 países de la Unión Europea. En Revista Latina de Comunicación Social. https://doi.org/10.4185/RLCS-2017-1218

Garrett, J. J. (2011). The elements of user experience: User-centered design for the web and beyond (2nd ed.). New Riders.

Gough, D., Oliver, S., \& Thomas, J. (2017). An introduction to systematic reviews (2nd ed.). SAGE Publications Ltd. https://uk.sagepub.com/en-gb/eur/an-introduction-to-systematicreviews/book 245742

Grant, M. J., \& Booth, A. (2009). A typology of reviews: an analysis of 14 review types and associated methodologies. Health Information \& Libraries Journal, 26(2), 91-108. https://doi.org/10.1111/j.1471-1842.2009.00848.x

Guallar, J., Codina, L., Freixa, P., \& Pérez-Montoro, M. (2020). Desinformación, bulos, curación y verificación. Revisión de estudios en Iberoamérica 2017-2020. Telos Revista de Estudios Interdisciplinarios en Ciencias Sociales, 22(3), 595-613. https://doi.org/10.36390/telos223.09

Guallar, J., Rovira, C., \& Ruiz, S. (2010). Multimedialidad en la prensa digital. Elementos multimedia y sistemas de recuperación en los principales diarios digitales españoles. Profesional de la Información, 19(6), 620-631. https://doi.org/10.3145/epi.2010.nov.08

Hasan, L., \& Abuelrub, E. (2011). Assessing the quality of web sites. Applied Computing and Informatics, 9(1), 11-29. https://doi.org/10.1016/j.aci.2009.03.001

Hernández Soriano, F. (2015). E-SQ-MEDIA, creación e implementación de una escala de medición de la calidad percibida en cibermedios [Universitat de Lleida]. En TDX (Tesis Doctorals en Xarxa). http://hdl.handle.net/10803/458999

Hernández Soriano, F., \& Cristóbal Fransi, E. (2013). Estado de la cuestión y nuevas perspectivas en la evaluación de la calidad percibida en los cibermedios. Historia y Comunicación Social, 18(0), 15-27. https://doi.org/10.5209/rev_HICS.2013.v18.44222

Hernández Soriano, F., \& Cristóbal Fransi, E. (2014). Evaluación de las herramientas Web 2.0 en el periodismo digital: el caso de VilaWeb. Historia y Comunicación Social, 19(0). https://doi.org/10.5209/rev_HICS.2014.v19.44942

Hernández Soriano, F., \& Cristóbal Fransi, E. (2016). La interacción como factor determinante de la calidad de un cibermedio. Opción: Revista de Ciencias Humanas y Sociales, 7(32), 848-864. http://repositori.udl.cat/handle/10459.1/59065

Iglesias-García, M., \& González-Díaz, C. (2012). Análisis de la calidad de la web del ciberdiario VilaWeb. Hipertext.net: Revista Académica sobre Documentación Digital y Comunicación Interactiva, 10, 20. https://www.upf.edu/hipertextnet/numero-10/vilaweb-analisis-calidad-webciberdiario.html

Internet Live Stats. (2021). Internet Usage \& Social Media Statistics. http://www.internetlivestats.com/ 
ISO. (2018). ISO 9241-11:2018 Ergonomics of human-system interaction - Part 11: Usability: Definitions and concepts (p. 32). International Organization for Standardization [ISO].

Jeong, W., \& Jung Han, H. (2012). Usability study on newspaper mobile websites. OCLC Systems \& Services: International digital library perspectives, 28(4), 180-198. https://doi.org/10.1108/10650751211279120

Jiménez Iglesias, L. (2018). Usabilidad para dispositivos móviles basada en la evidencia: los cibermedios españoles [Universitat de Barcelona]. En TDX (Tesis Doctorals en Xarxa). http://www.tdx.cat/handle/10803/463040

Jowkar, A., \& Didegah, F. (2010). Evaluating Iranian newspapers' web sites using correspondence analysis. Library Hi Tech, 28(1), 119-130. https://doi.org/10.1108/07378831011026733

Krug, S. (2014). Don't make me think, revisited: A common sense approach to web and mobile usability (New Riders (ed.); 3rd ed.). Pearson Education. http://sensible.com/dmmt.html

Ksiazek, T. B. (2018). Commenting on the news: Explaining the degree and quality of user comments on news websites. Journalism Studies, 19(5), 650-673. https://doi.org/10.1080/1461670X.2016.1209977

Langer, J., \& Zeiller, M. (2017, noviembre 30). Evaluation of the User Experience of Interactive Infographics in Online Newspapers. 10th Forum Media Technology 2017. https://people.fhburgenland.at/handle/20.500.11790/961

Leavitt, M. O., \& Shneiderman, B. (2006). Research-based web design \& usability guidelines (U.S. Department of Health \& Human Services (ed.); 2nd ed.). https://guidelines.usability.gov/

Limia Fernández, M., Toural Bran, C., \& López García, X. (2013). Interactividad y participación en los cibermedios: una propuesta metodológica para la elaboración, registro y análisis de datos. $2^{\circ}$ Congreso Nacional sobre Metodología de la Investigación en Comunicación: Investigar la Comunicación hoy. Revisión de políticas científicas y aportaciones metodológicas, 187-204. http://uvadoc.uva.es/handle/10324/3030

Linares, J., Codina, L., \& Freixa, P. (2015). Tendencias en cibermedios: análisis de la interactividad. http://hdl.handle.net/10230/24636

Linares, J., Codina, L., \& Pedraza-Jiménez, R. (2015). Interactividad en cibermedios: Propuesta nuclear de protocolo de análisis. http://hdl.handle.net/10230/23167

Linares, J., Codina, L., Vàllez Letrado, M., \& Rodríguez Martínez, R. (2016). Interactividad, buscabilidad y visibilidad en cibermedios: sistema de análisis y resultados (Serie Editorial Digidoc. Proyecto Audiencias Activas). https://repositori.upf.edu/handle/10230/26280

Linares, J., Codina, L., Vàllez, M., \& Rodríguez-Martínez, R. (2016). Interactividad, buscabilidad y visibilidad en cibermedios: sistema de análisis y resultados (Serie Editorial Digidoc. Proyecto Audiencias Activas). http://hdl.handle.net/10230/26280 
RLCS, Revista Latina de Comunicación Social, 80, 39-63

[Investigación] DOI: 10.4185/RLCS-2022-1515| ISSN 1138-5820| Año 2022

López García, G. (Ed. . (2008). Los cibermedios valencianos: Cartografía, características y contenidos. Servei de Publicacions de la Universitat de València. https://libros.metabiblioteca.org/handle/001/319

Lopezosa, C., \& Codina, L. (2018). Análisis de posicionamiento en medios de comunicación con herramientas SEO: cobertura informativa de los premios Oscar 2017 (Serie Editorial DigiDoc EPI). http://hdl.handle.net/10230/33632

Lopezosa, C., Codina, L., \& Gonzalo-Penela, C. (2019). Off-page SEO and link building: General strategies and authority transfer in the digital news media. Profesional de la Información, 28(1). https://doi.org/10.3145/epi.2019.ene.07

Lopezosa, C., Codina, L., López-García, G., \& Corbella-Cordomi, J.-M. (2020). Mapa de visibilidad y posicionamiento en buscadores de los principales grupos mediáticos españoles. Profesional de la Información, 29(2). https://doi.org/10.3145/epi.2020.mar.03

Lopezosa, C., García-Carretero, L., Gonzalo-Penela, C., \& Codina, L. (2020). Sistema de análisis (AVCIS) de la visibilidad en cibermedios mediante indicadores SEO. En K. Meso Ayerdi, S. Peña Fernández, \& A. Larrondo Ureta (Eds.), XI Congreso Internacional de Ciberperiodismo (Bilbao): Nuevos actores y estrategias en el espacio mediático compartido (pp. 185-204). Universidad del País Vasco. https://dialnet.unirioja.es/servlet/articulo?codigo=7433485

Lopezosa, C., Iglesias-García, M., González-Díaz, C., \& Codina, L. (2020). Experiencia de búsqueda en cibermedios: análisis comparativo de diarios nativos digitales. Revista Española de Documentación Científica, 43(1), e254. https://doi.org/10.3989/REDC.2020.1.1677

Mariage, C., \& Vanderdonckt, J. (2001). A comparative usability study of electronic newspapers. En Tools for Working with Guidelines (pp. 325-337). Springer London. https://doi.org/10.1007/978$\underline{1-4471-0279-3 \_31}$

Martín-Martín, A., Orduna-Malea, E., Thelwall, M., \& Delgado López-Cózar, E. (2018). Google Scholar, Web of Science, and Scopus: A systematic comparison of citations in 252 subject categories. Journal of Informetrics, 12(4), 1160-1177. https://doi.org/10.1016/j.joi.2018.09.002

Martín-Sanromán, J.-R., Suárez Carballo, F., \& Zambrano, J.-C. (2019). La experiencia de usuario en los productos periodísticos: un enfoque desde el diseño centrado en el lector-usuario. El Argonauta español, 16. https://doi.org/10.4000/ARGONAUTA.3540

Martins, G. L. (2012). Protocolo de qualidade para conteúdos em ciberjornalismo: estudo comparativo Espanha e Brasil em portais de notícias de referência. $10^{\circ}$ Encontro Nacional de Pesquisadores em Jornalismo - Associação Brasileira de Pesquisadores em Jornalismo (SBPJor), 1-17. https://www.researchgate.net/publication/236647194

Masip, P., Díaz-Noci, J., Domingo, D., Micó-Sanz, J.-L., \& Salaverría, R. (2010). Investigación internacional sobre ciberperiodismo: hipertexto, interactividad, multimedia y convergencia. El Profesional de la Información, 19(6), 568-576. https://doi.org/10.3145/epi.2010.nov.02

Mauri-Ríos, M., \& Ramon-Vegas, X. (2015). Nuevos sistemas de rendición de cuentas de la información periodística. Exploración del escenario online español. Profesional de la Información, 24(4), 380. https://doi.org/10.3145/epi.2015.jul.04 
Mogollón, H., Acevedo, A., \& Ramírez, J. (2006). Cibermedios venezolanos: Bajo la lupa de la usabilidad. Comunicación: estudios venezolanos de comunicación, 136(4), 36-43. https://dialnet.unirioja.es/servlet/articulo?codigo=2294183

Morales-Vargas, A., Pedraza-Jiménez, R., \& Codina, L. (2020). Website quality: An analysis of scientific production. Profesional de la Información, 29(5), e290508. https://doi.org/10.3145/epi.2020.sep.08

Newman, N., Fletcher, R., Kalogeropoulos, A., Levy, D. A. L., \& Nielsen, R. K. (2018). Reuters Institute Digital News Report 2018 (Vol. 1). https://www.digitalnewsreport.org

Newman, N., Fletcher, R., Schulz, A., Andi, S., Robertson, C. T., \& Nielsen, R. K. (2021). Digital News Report 2021. https://reutersinstitute.politics.ox.ac.uk/digital-news-report/2021

Nielsen, J. (2000). Designing web usability. New Riders.

Nielsen, J. (2020). 10 usability heuristics for user interface design. Nielsen Norman Group. https://www.nngroup.com/articles/ten-usability-heuristics/

Nunes, S., Almeida, A., Giesteira, B., Pinto, F., \& Oliveira, P. (2007). Interface evaluation of Portuguese and international news websites. MCCSIS 2007 - IADIS Multi Conference on Computer Science and Information Systems - Proceedings of Intelligent Systems and Agents 2007, Interfaces and Human Computer Interaction 2007, Computer Graphics and Visualization 2007, 109-116.

O’Brien, H. L., \& Lebow, M. (2013). Mixed-methods approach to measuring user experience in online news interactions. Journal of the American Society for Information Science and Technology, 64(8), 1543-1556. https://doi.org/10.1002/asi.22871

Odriozola Chéné, J., Aguirre Mayorga, C., \& Bernal Suárez, J. D. (2017). Condicionantes en la calidad de los contenidos de los cibermedios ecuatorianos: convergencia periodística, agenda temática e inmediatez. Estudios sobre el Mensaje Periodístico, 22(2), 1103-1121. https://doi.org/10.5209/ESMP.54254

Olsina, L., Covella, G., \& Rossi, G. (2006). Web quality. En E. Mendes \& N. Mosley (Eds.), Web Engineering (pp. 109-142). Springer Berlin Heidelberg. https://doi.org/10.1007/3-540-28218-1_4

Omidvar, A., Pourmodheji, H., An, A., \& Edall, G. (2020). Learning to Determine the Quality of News Headlines. Proceedings of the 12th International Conference on Agents and Artificial Intelligence, 1, 401-409. https://doi.org/10.5220/0009367504010409

Palacios, M., \& Díaz Noci, J. (2009). Online journalism: research methods. A multidisciplinary approach in comparative perspective. Servicio Editorial de la Universidad del País Vasco/Euskal Herriko Unibertsitatearen Argitalpen Zerbitzua.

Pedraza-Jiménez, R., Codina, L., \& Guallar, J. (2016). Calidad en sitios web: Método de análisis general, e-commerce, imágenes, hemerotecas y turismo. Editorial UOC. 
Pribeanu, C. (2009). A usability assistant for the heuristic evaluation of interactive systems. Studies in Informatics and Control, 18(4), 355-362.

Rabaya Toma, T., Mahmud, I., Emran Hossain, M., Jahan, N., Ramayah, T., \& Jayapal, P. (2018). To Read or Not to Read: Modeling Online Newspaper Reading Satisfaction and Its Impact on Revisit Intention and Word-Of-Mouth. Interdisciplinary Journal of Information, Knowledge, and Management, 13, 337-359. https://doi.org/10.28945/4118

Rekik, R., Kallel, I., Casillas, J., \& Alimi, A. M. (2018). Assessing web sites quality: A systematic literature review by text and association rules mining. International Journal of Information Management, 38(1), 201-216. https://doi.org/10.1016/J.IJINFOMGT.2017.06.007

Rivas-de-Roca, R., Caro-González, F. J., \& García-Gordillo, M. (2020). Indicadores transnacionales de calidad informativa basados en la experiencia de periodistas locales: estudios de caso en medios digitales de Alemania, España y Reino Unido. Congreso Internacional de la Asociación Española de Investigación de la Comunicación, 39-50. https://doi.org/10.3145/AE-ICepi.2020.e03

Rodríguez-Martínez, R., Codina, L., \& Pedraza-Jiménez, R. (2010). Cibermedios y web 2.0: modelo de análisis y resultados de aplicación. Profesional de la Información, 19(1), 35-44. https://doi.org/10.3145/epi.2010.ene.05

Rodríguez-Martínez, R., Codina, L., \& Pedraza-Jiménez, R. (2012). Indicadores para la evaluación de la calidad en cibermedios: análisis de la interacción y de la adopción de la Web 2.0. Revista española de Documentación Científica, 35(1), 61-93. https://doi.org/10.3989/redc.2012.1.858

Romero-Rodríguez, L. M., De-Casas-Moreno, P., \& Torres-Toukoumidis, Á. (2016). Dimensiones e indicadores de la calidad informativa en los medios digitales. Comunicar, 24(49), 91-100. https://doi.org/10.3916/C49-2016-09

Rosa, J. M., \& Veras, M. (2013). Avaliação heurística de usabilidade em jornais online: estudo de caso em dois sites. Perspectivas em Ciência da Informação, 18(1), 138-157. https://doi.org/10.1590/S1413-99362013000100010

Rosala, M., \& Krause, R. (2020). User Experience Careers: What a Career in UX Looks Like Today. https://www.nngroup.com/reports

Rosenfeld, L., Morville, P., \& Arango, J. (2015). Information Architecture: For the web and beyond (4th ed.). O’Reilly Media.

Rubin, J., \& Chisnell, D. (2008). Handbook of usability testing: How to plan, design, and conduct effective tests. Wiley.

Said-Hung, E., \& Arcila-Calderón, C. (2011a). Hacia un índice de medición del desarrollo de los cibermedios. Actas de la $V$ Conferencia ACORN-REDECOM. http://www.prensaescrita.com/america/colombia.php

Said-Hung, E., \& Arcila-Calderón, C. (2011b). Los cibermedios en América Latina y la Web 2.0. Comunicar, 19(37), 125-131. https://doi.org/10.3916/C37-2011-03-04 
RLCS, Revista Latina de Comunicación Social, 80, 39-63

[Investigación] DOI: 10.4185/RLCS-2022-1515| ISSN 1138-5820|

Salas Hernández, T. U., Hernández Estrada, Ó., \& Realyvázquez Quintana, J. A. (2018). Factores de accesibilidad y calidad en cibermedios mexicanos: caso Chihuahua. Ámbitos. Revista Internacional de Comunicación, 40. https://idus.us.es/handle/11441/71913

Salaverría, R. (2017). Tipología de los cibermedios periodísticos: bases teóricas para su clasificación. Revista Mediterránea de Comunicación, 8(1). https://doi.org/10.14198/medcom2017.8.1.2

Salaverría, R. (2019). Periodismo digital: 25 años de investigación. Artículo de revisión. Profesional de la Información, 28(1), 1699-2407. https://doi.org/10.3145/epi.2019.ene.01

Salaverría, R., Buslón, N., López-Pan, F., León, B., López-Goñi, I., \& Erviti, M.-C. (2020). Desinformación en tiempos de pandemia: tipología de los bulos sobre la Covid-19. Profesional de la Información, 29(3). https://doi.org/10.3145/epi.2020.may.15

Salaverría, R., Cores, R., Díaz-Noci, J., Meso Ayerdi, K., \& Larrondo Ureta, A. (2004). Evaluación de los ciberdiarios en las comunidades vasca y navarra. Communication \& Society, 17(1), 161192. http://www.comunicacionysociedad.com/es/articulo.php?art_id=78

Sanabre Vives, C. (2015). Un modelo para el análisis y concepción sitios web: El WebSite Canvas Model aplicado a Eldiario.es. Hipertext.net: Revista Académica sobre Documentación Digital y Comunicación Interactiva, 13, 19. https://doi.org/10.2436/20.8050.01.20

Sánchez-González, M., \& Alonso, J. (2012). Propuesta metodológica para el análisis de las tecnologías de participación en cibermedios. Revista Latina de Comunicación Social (67). https://doi.org/10.4185/RLCS-067-951-148-178

Sauro, J. (2010). A practical guide to measuring usability: Quantifying the usability of websites and software. CreateSpace.

Sauro, J., \& Lewis, J. R. (2016). Quantifying the user experience: practical statistics for user research (Second ed.). Elsevier / Morgan Kaufmann.

Scharkow, M. (2013). Thematic content analysis using supervised machine learning: An empirical evaluation using German online news. Quality \& Quantity, 47(2), 761-773. https://doi.org/10.1007/s11135-011-9545-7

Serm, T. C., Blanchfield, P., \& Su, K. S. D. (2006). Mobile newspaper development framework: Guidelines for newspaper companies for creating usable mobile news portals. 2006 International Conference on Computing \& Informatics, 1-8. https://doi.org/10.1109/ICOCI.2006.5276443

Shneiderman, B. (2016). The eight golden rules of interface design. Department of Computer Science, University of Maryland. https://www.cs.umd.edu/users/ben/goldenrules.html

The Trust Project. (2020). The Trust Indicators and their Attributes. Markkula Center for Applied Ethics, Santa Clara University. https://thetrustproject.org/

Thelwall, M., \& Kousha, K. (2015). Web indicators for research evaluation. Part 2: Social media metrics. Profesional de La Información, 24(5), 607-620. https://doi.org/10.3145/epi.2015.sep.09 
Tognazzini, B. (2014). First principles of interaction design (revised and expanded). En Ask TOG: Interaction design solutions for the real world. https://asktog.com/atc/principles-of-interaction$\underline{\text { design/ }}$

Tullis, T., \& Albert, W. (2013). Measuring the user experience: Collecting, analyzing, and presenting usability metrics (2nd ed.). Morgan Kaufmann. https://www.sciencedirect.com/book/9780124157811/measuring-the-user-experience

Túñez López, M., \& Nogueira, A. G. (2017). Infographics as a mnemonic structure: Analysis of the informative and identity components of infographic online compositions in iberic newspapers. Communication and Society, 30(1), 147-164. https://doi.org/10.15581/003.30.1.147-164

Ugras, T., Gülseçen, S., Çubukçu, C., Erdoğmuş, İ. İ., Gashi, V., \& Bedir, M. (2016). Research trends in web site usability: A systematic review. En A. Marcus (Ed.), Design, User Experience, and Usability: Design Thinking and Methods (pp. 517-528). Springer International Publishing. https://link.springer.com/chapter/10.1007\%2F978-3-319-40409-7_49

Unesco. (2019). UNESCO's Internet universality indicators: A framework for assessing internet development. United Nations Educational, Scientific and Cultural Organization. https://unesdoc.unesco.org/ark:/48223/pf0000367617

Unión Europea. (2016). Reglamento general de protección de datos. Diario Oficial de la Unión Europea. https://europa.eu/!TJ96Hd

Usability.gov. (2013). Heuristic evaluations and expert reviews. Department of Health and Human Services. https://www.usability.gov/how-to-and-tools/methods/heuristic-evaluation.html

Yu, N., \& Kong, J. (2016). User experience with web browsing on small screens: Experimental investigations of mobile-page interface design and homepage design for news websites. Information Sciences, 330, 427-443. https://doi.org/10.1016/j.ins.2015.06.004

Zambarbieri, D., Carniglia, E., \& Robino, C. (2008). Eye tracking analysis in reading online newspapers. Journal of Eye Movement Research, 2(4). https://doi.org/10.16910/jemr.2.4.7

\section{AUTORES:}

\section{Alejandro Morales Vargas}

Doctor en Comunicación en la Universitat Pompeu Fabra (UPF). Profesor Asistente del Instituto de la Comunicación e Imagen (ICEI) de la Universidad de Chile. Colaborador del Grupo de Investigación de Documentación Digital y Comunicación Interactiva (DigiDoc) de la UPF. Periodista y Licenciado en Comunicación Social por la Universidad de Chile; Máster en Gestión de Contenidos Digitales por la Universitat de Barcelona. Se ha desempeñado como editor de sitios web, profesor universitario en grado y postgrado, evaluador de proyectos y consultor en arquitectura de información. Fue creador del Diplomado en Periodismo Digital y Gestión de Medios en Internet del ICEI y jefe de Medios Digitales en la Dirección de Servicios de Información y Bibliotecas (SISIB), ambos en la U. de Chile.

Índice H: 4 (agosto 2021)

Orcid ID: https://orcid.org/0000-0002-5681-8683 
RLCS, Revista Latina de Comunicación Social, 80, 39-63

[Investigación] DOI: 10.4185/RLCS-2022-1515| ISSN 1138-5820| Año 2022

Google Scholar: https://scholar.google.com/citations?user=qxsUqxoAAAAJ

ResearchGate: https://www.researchgate.net/profile/Alejandro_Morales_Vargas

Scopus ID: https://www.scopus.com/authid/detail.uri?authorId=57222327924

Academia.edu: https://uchile.academia.edu/AlejandroMorales Vargas

Publons: https://publons.com/researcher/3399627/alejandro-morales-vargas/

Dialnet: https://dialnet.unirioja.es/servlet/autor?codigo $=3789777$

Página web: $\underline{\text { http://uchile.cl/c46659 }}$

\section{Rafael Pedraza-Jiménez}

Doctor en Información y Documentación por la Universidad de Barcelona. Profesor Agregado Serra Húnter en el Departamento de Comunicación de la Universitat Pompeu Fabra, donde también es Secretario de la Facultad de Comunicación y miembro investigador del Grupo de Investigación de Documentación Digital y Comunicación Interactiva (DigiDoc). Imparte docencia en los estudios de Periodismo y Comunicación Audiovisual de la UPF. Además, es profesor en diversos másteres y postgrados. Ha publicado diversos artículos en revistas de impacto a nivel internacional, y algunas de sus principales líneas de trabajo son: calidad web, cibermedios, recuperación de información, estudios de la información y estudios de comunicación.

Índice H: 19 (agosto 2021)

Orcid ID: https://orcid.org/0000-0002-6918-6910

Google Scholar: https://scholar.google.com/citations?user=aMM9QaYAAAAJ\&hl

ResearchGate: https://www.researchgate.net/profile/Rafael_Pedraza-Jimenez

Scopus ID: $\underline{\text { https://www.scopus.com/authid/detail.uri?authorId=55890005200 }}$

Academia.edu: $\underline{\text { https://upf.academia.edu/RafaelPedrazaJimenez }}$

Publons: https://publons.com/researcher/2890290/rafael-pedraza-jimenez/

Dialnet: https://dialnet.unirioja.es/servlet/autor?codigo=1986796

\section{Lluís Codina}

Doctor en Ciencias de la Información por la Universitat Autònoma de Barcelona. Profesor titular del Departamento de Comunicación de la Universitat Pompeu Fabra (UPF). Coordinador de la Unidad de Investigación en Periodismo y Documentación Digital y miembro investigador del Grupo DigiDoc. Coordinador del Master Universitario en Comunicación Social (MUCS). Docente de la Facultad de Comunicación, en los grados de Periodismo y de Comunicación Audiovisual. Docente de los Masters Universitarios Online en Documentación Digital y en Buscadores de la Barcelona School of Management de la UPF. Codirector del Observatorio de Cibermedios. Coinvestigador principal del proyecto «Narración interactiva y visibilidad digital en el documental interactivo y el periodismo estructurado» (MICINN, España/FEDER, UE).

Índice H: 33 (agosto 2021)

Orcid ID: https://orcid.org/0000-0001-7020-1631

Google Scholar: https://scholar.google.com/citations?user=89k7vMMAAAAJ

ResearchGate: https://www.researchgate.net/profile/Lluis_Codina2

Scopus ID: https://www.scopus.com/authid/detail.uri?authorId=23392068000

Academia.edu: https://upf.academia.edu/lluiscodina

Publons: https://publons.com/researcher/1174214/lluis-codina/

Dialnet: https://dialnet.unirioja.es/servlet/autor?codigo=259661

Sitio web: https://www.lluiscodina.com/ 